\title{
Tratamiento quirúrgico de la enfermedad venosa crónica en octogenarios
}

\section{Surgical treatment of chronic venous insufficiency in octogenarians}

\author{
Rodrigo Garza-Herrera* \\ Centro Médico Healthy Steps, Morelia, Michoacán, México
}

\begin{abstract}
Resumen
La población de edad avanzada a nivel mundial se encuentra en constante crecimiento, con alrededor de 125 millones de personas mayores de 80 años de edad. La prevalencia de enfermedad venosa crónica aumenta con la edad, encontrando asociación entre edad avanzada y estadios clínicos Clínica, Etiología, Anatomía, Fisiopatología (CEAP) más avanzados. Históricamente, la tendencia de atención a los pacientes en este grupo de edad ha sido el tratamiento médico conservador, sin embargo, la edad es una limitante para el uso de terapia compresiva, ya que aumenta el tiempo para lograr el cierre de úlceras varicosas, lo que provoca un incremento en los costos. Tanto la cirugía abierta como las técnicas de ablación endovenosa han demostrado seguridad, éxito técnico y satisfacción en el tratamiento de los pacientes octogenarios, con mayor número de complicaciones en técnicas abiertas que en técnicas de ablación endovenosa. La edad no debe ser una limitante para ofrecer procedimientos quirúrgicos en dichos pacientes.
\end{abstract}

Palabras clave: Edad avanzada. Enfermedad venosa crónica. Ablación endovenosa. Octogenarios. Nonagenarios. Centenarios.

\begin{abstract}
The world's elderly population is constantly growing, with approximately 125 million people above the age 80 . The prevalence of chronic venous disease increases with age, finding association between older age and more advanced CEAP clinical stages. Historically the tendency of patient care in this age group has been conservative medical treatment, however, age is an important limitation in the adequate use of compression therapy, with increased total cost of treatment and longer time to achieve wound closure in varicose ulcers. Both open surgery and endovenous ablation techniques have demonstrated safety, technical success and satisfaction in the treatment of octogenarian, nonagenarian and centenarian patients, with a greater number of complications for open techniques than for endovenous ablation. Age should not be a contraindication for surgical procedures.
\end{abstract}

Key words: Elderly. Chronic venous insufficiency. Endovenous ablation. Octogenarians. Nonagenarians. Centenarians.

Correspondencia:

*Rodrigo Garza-Herrera

E-mail: garzavascular@gmail.com
Disponible en internet: 26-10-2020

Rev Mex Angiol. 2020;48(3):96-99

www.RMAngiologia.com bajo la licencia CC BY-NC-ND license (http://creativecommons.org/licenses/by-nc-nd/4.0/). 


\section{Introducción}

La expectativa de vida en la población mundial ha aumentado, por lo que los pacientes de edad avanzada actualmente tienen más de 80 años. La población mayor de 60 años representó el 12\% de la población en el año 2015, para 2020 se reporta que existen más personas mayores de 60 años que niños menores de 5 años, con estimación de que dicho grupo etario aumente al $22 \%$ de la población para el año 2050. Actualmente se estima que en el mundo existen 125 millones de personas con más de 80 años, cifra que puede aumentar a 434 millones para el año 2050, de los cuales, el $80 \%$ vivirán en países de ingresos bajos a medianos ${ }^{1}$. El objetivo de esta revisión es conocer el comportamiento de la patología venosa en dichos grupos etarios, así como la seguridad y eficacia de su tratamiento, lo cual nos servirá para esclarecer y dictaminar las directrices del tratamiento a tomar en los años venideros. Enfrentarnos a este tipo de pacientes se vuelve un reto en la práctica médica diaria, además del aumento en las comorbilidades de este grupo, la prevalencia de la úlcera varicosa tiene un crecimiento exponencial con la edad, debido a falla de la bomba muscular de la pantorrilla, asociada a artritis, enfermedades neurológicas, rigidez en la articulación del tobillo o alteración en el drenaje linfático². El estudio Basilea demostró un aumento significativo en la prevalencia de enfermedad venosa de acuerdo con la edad, que representa el $4 \%$ en pacientes de 30 años e incrementa al $35 \%$ en pacientes de 70 años $^{3}$, y un aumento en la presencia de insuficiencia venosa funcional, que alcanza un $24 \%$ en pacientes entre 65 y 69 años de edad, pero un incremento reportado hasta el $83 \%$ en pacientes mayores de 80 años de edad ${ }^{4}$. Esto se traduce en que la edad aumenta el odds ratio 2.42 para venas varicosas y 4.85 para enfermedad venosa crónica en pacientes mayores de 65 años, cifra que alcanza 23.3 en pacientes entre 70-79 años de edad 5 . Ante estos datos, entendemos que la edad y la enfermedad venosa crónica tienen una asociación directa para estadios clínicos más avanzados. Dicha asociación también se puede ver afectada por la tendencia inicial a ofrecer manejo conservador a estos pacientes o a un retraso en el referimiento al especialista por parte de los médicos de primer contacto, por lo que hasta el 53.6-56\% de los pacientes mayores de 80 años se encuentran en estadio clínico C4 o mayor ${ }^{6,7}$. El ofrecer un tratamiento efectivo y seguro a este grupo de pacientes se ha convertido en un tema de relevancia actual y hacia el panorama futuro.
El reto principal de atender a pacientes mayores de 80 años con presencia de úlcera varicosa es que el tiempo promedio para lograr un adecuado cierre de la herida es más prolongado, el $74 \%$ de las heridas cierran en un periodo de 6 meses $^{2}$.

\section{Tratamiento conservador}

Una de las mayores limitantes del tratamiento conservador es la dificultad de colocación de terapias de compresión, especialmente vendajes y medias compresivas, donde el paciente de edad avanzada requiere habitualmente de ayuda para su colocación o de dispositivos adicionales que aumentan el costo en el tratamiento. El beneficio en la terapia compresiva para aumentar la función de la bomba muscular y el drenaje linfático y reducir el edema linfático se observa en compresiones por arriba de $30 \mathrm{mmHg}$, compresión habitualmente poco tolerada por pacientes de edad avanzada, con el $18 \%$ de los pacientes rechazando todas las opciones de terapia compresiva disponibles $^{2,4}$ y el $40 \%$ sin apego al tratamiento ${ }^{8}$. El costo a largo plazo para lograr el cierre de una úlcera varicosa es mayor para el tratamiento conservador en comparación con las técnicas de cirugía por ablación $n^{9,10}$.

\section{Técnica quirúrgica abierta}

La técnica de cirugía abierta ha demostrado la misma seguridad en pacientes mayores de 65 años que en pacientes jóvenes ${ }^{11,12}$. Un metaanálisis reportado por Gang $^{13}$, et al. encuentra que la recurrencia clínica referida por el paciente puede ser hasta del 33\% a 2 años utilizando estas técnicas y la tasa de complicaciones puede ascender hasta el 18 a 20\%. Así mismo se asocia a mayor pérdida sanguínea y aumento en el tiempo quirúrgico en comparación con las técnicas de ablación. Otra limitante para la técnica abierta es el alto rechazo de los pacientes, con el $17.7 \%$ de asociación a trastorno de ansiedad asociado a la indicación de cirugía ${ }^{14}$.

\section{Técnicas endovenosas}

Distintos estudios han demostrado eficacia y seguridad con técnicas de termoablación en pacientes mayores de 80 años de edad $6,7,15-18$. Está descrito que, en pacientes mayores de 65 años de edad, la probabilidad de desarrollar trombosis inducida por energía térmica (EHIT) es mayor ${ }^{16,17}$. Por ello, Kibrik, et al. ${ }^{18}$ estudiaron específicamente la población mayor de 80 años de edad, y establecieron que la edad es un factor determinante 
Tabla 1. Ventajas y desventajas de las principales opciones para el tratamiento de la enfermedad venosa crónica sintomática en pacientes mayores a 80 años de edad

\begin{tabular}{|l|l|l|}
\hline Técnica & Ventajas & Desventajas \\
\hline Termoablación & $\begin{array}{l}\text { Éxito técnico } 90 \% \\
\text { Satisfacción } 98 \% \\
\text { Mejor evidencia relación costo-beneficio } \\
\text { Procedimiento ambulatorio. }\end{array}$ & $\begin{array}{l}\text { Probabilidad de EHIT 8.8\% } \\
\text { Recurrencia hasta } 42 \% \text { a } 5 \text { años. }\end{array}$ \\
\hline Cirugía abierta & $\begin{array}{l}\text { Misma seguridad que en pacientes jóvenes. } \\
\text { Buena satisfacción por paciente. } \\
\text { Eścnica con menor probabilidad de reintervención a } 5 \text { años. }\end{array}$ & $\begin{array}{l}\text { Complicaciones hasta } 18-20 \% \\
\text { Recurrencia hasta } 46 \% \text { a } 5 \text { años. } \\
\text { Hasta } 17.7 \% \text { de rechazo al procedimiento. }\end{array}$ \\
\hline Conservador & $\begin{array}{l}\text { Buena satisfacción por paciente. } \\
\text { Procedimiento ambulatorio. }\end{array}$ & $\begin{array}{l}\text { Recurrencia hasta } 53 \% \text { a } 5 \text { años. } \\
\text { Técnica con mayor probabilidad de } \\
\text { reintervención. }\end{array}$ \\
\hline & $\begin{array}{l}\text { Alta aceptación por paciente. } \\
\text { Efectiva con compresión }>30 \mathrm{mmHg} .\end{array}$ & $\begin{array}{l}\text { Hasta } 40 \% \text { de falta de apego al tratamiento } \\
\text { compresivo. } \\
\text { Costo a largo plazo más elevado para cierre de } \\
\text { úlcera varicosa. }\end{array}$ \\
\hline
\end{tabular}

para la presencia de EHIT, siendo especialmente elevada en pacientes de más de 90 años, quienes también presentaron cifras más elevadas de recanalización, teniendo diferencia significativa con los menores de 80 años. Dicha relación fue significativa en los pacientes tratados mediante radiofrecuencia (RFA), asociación no vista con ablación láser (EVLA). En la población estudiada, se detectó que el $73.7 \%$ de los casos descritos fueron EHIT tipo 1, el $25.3 \%$ fueron tipo 2 y solo el $0.5 \%$ fueron tipos 3-4. Dichos resultados son importantes, ya que el tratamiento de EHIT tipos 1 y 2 se realiza mediante vigilancia con ultrasonido y, en casos determinados, anticoagulante oral directo ${ }^{19}$, por lo que el riesgo relativo de EHIT en pacientes tratados con RFA mayores de 80 años no se asocia a complicaciones que pongan en riesgo la calidad de vida del paciente.

La RFA demostró mayor beneficio en disminución del dolor postoperatorio, marcha temprana y menor perforación venosa, resultando en menor equimosis y dolor en comparación con EVLA ${ }^{15}$, por lo que el riesgo relativo de EHIT en RFA, donde el $99 \%$ de los eventos se limitaron tipo 1 y 2, puede ser asumido en pacientes con poca tolerancia al dolor o con alteraciones de hipersensibilidad a múltiples fármacos.

Los resultados sobre la terapia de ablación en safena mayor en pacientes mayores de 80 años muestran que existe mejoría en los scores de severidad tanto clínico como en calidad de vida manifestado por los pacientes. Se ha reportado éxito técnico en más del $90 \%$ de los casos y satisfacción referida por el paciente en el $98 \%$ de los casos en un periodo de 24 meses $^{15-17}$. La causa del alto éxito obtenido se debe a que habitualmente los pacientes mayores de 80 años de edad cursan con enfermedad troncal, con poca necesidad de flebectomías asociadas, lo que se traduce en éxito para el tratamiento primario en la úlcera varicosa ${ }^{6,15}$.

No se han reportado estudios que demuestren la eficacia y seguridad de las técnicas no térmicas, no tumescentes en pacientes octogenarios, nonagenarios y centenarios. Tomando en cuenta que dichos métodos han demostrado equivalencia con las técnicas de ablación endovenosa y superioridad a la escleroterapia guiada por ultrasonido ${ }^{20}$, podríamos contemplarlas como una posibilidad en el tratamiento de este tipo de pacientes.

\section{Escleroterapia de safena mayor}

En septiembre de 2019 se publicó el estudio CLASS, un estudio prospectivo aleatorizado que compara técnica con método de ablación, técnicas abiertas y escleroterapia de safena mayor, y encontró que el beneficio a 5 años favorece a la ablación quirúrgica, después a la cirugía abierta y, por último, la escleroterapia con espuma. Se demostró también que la escleroterapia de la safena mayor es la técnica con mayor probabilidad de reintervención, hasta en un $11 \%$ de los pacientes ${ }^{21}$. Además, el tratamiento con espuma no es recomendable por presencia de mayor rigidez venosa en pacientes añosos en comparación con jóvenes, con menor tendencia al vasoespasmo, así como necesidad de mayor número de sesiones de tratamiento y menor efectividad, especialmente en safenas mayores de $6 \mathrm{~mm}$; así como menor necesidad de analgesia con láser $1480 \mathrm{~nm}$ que en pacientes tratados con esta técnica ${ }^{22}$.

En la tabla 1 se describen las principales ventajas y desventajas de las distintas técnicas disponibles para 
el tratamiento de la enfermedad venosa crónica sintomática en pacientes mayores de 80 años de edad.

\section{Conclusión}

Existe superioridad demostrada mediante técnicas de ablación en comparación a técnica de cirugía tradicional o manejo conservador para tratar estadios avanzados de enfermedad venosa crónica en pacientes mayores de 80 años, por lo que la edad no debe ser una limitante para ofertar un procedimiento endovenoso. Los resultados arrojados por el Vascular Quality Initiative Varicose Vein Registry (VQI VVR) nos permiten conocer más a este grupo de pacientes habitualmente no considerados para ensayos clínicos, por lo que, si bien los estudios mencionados se tratan de revisiones retrospectivas, nos pueden brindar información suficiente para considerar esta terapia en pacientes de edad avanzada.

\section{Agradecimientos}

Los autores agradecen a Dr. Carlos Arturo Hinojosa Becerril, Dr. Javier Eduardo Anaya Ayala.

\section{Conflicto de intereses}

Los autores declaran no tener ningún conflicto de intereses.

\section{Responsabilidades éticas}

Protección de personas y animales. Los autores declaran que para esta investigación no se han realizado experimentos en seres humanos ni en animales.

Confidencialidad de los datos. Los autores declaran que en este artículo no aparecen datos de pacientes.

Derecho a la privacidad y consentimiento informado. Los autores declaran que en este artículo no aparecen datos de pacientes.

\section{Bibliografía}

1. World Health Organization. Ageing and health. Disponible en: www.who. int/news-room/fact-sheets/detail/ageing-and- health. 5 February 2018.

2. Hansson C. Optimal Treatment of Venous (Stasis) Ulcers in Elderly Patients. Drugs Aging. 1994;5(5):323-34.

3. Callam MJ. Epidemiology of varicose veins. Br J Surg. 1994;81:167-73.

4. Suehiro K, Morikage N, Yamashita O, Harada T, Ueda K, Samura M, et al. Adherence to and Efficacy of Different Compression Methods for Treating Chronic Venous Insufficiency in the Elderly. Phlebology. 2016; 31(10):723-8.

5. Quarto G, Prophylactic GSV. Surgery in Elderly Candidates for Hip or Knee Arthroplasty. Open Med. 2016;11:471-6.

6. Sutzko D, Obi A, Kimball A, Smith M, Wakefield T, Osborne N. Clinical outcomes after varicose vein procedures in octogenarians within the Vascular Quality Initiative Varicose Vein Registry. J Vasc Surg Venous Lymphat Disord. 2018;6(4):464-70.

7. Hong KH, Spinedi L, Staub D, Diehm N, Holtz D, Broz P, et al. Safety and Efficacy of Outpatient Endovenous Laser Ablation in Patients 75 years and Older: A Propensity Score-matched Analysis. Swiss Med Wkly. 2019;149:w20083.

8. Sippel K, Seifert B, Hafner J. Donning Devices (Foot Slips and Frames) Enable Elderly People with Severe Chronic Venous Insufficiency to put on Compression Stockins. Eur J Vasc Endovasc Surg. 2015;49:221-29.

9. Marston WA, Carlin RE, Passman MA, Farber MA, Keagy BA. Healing rates and cost efficacy of outpatient compression treatment for leg ulcers associated with venous insuffi- ciency. J Vasc Surg. 1999;30:491-8.

10. Lin JC, Nerenz DR, Migliore P, Young R, Shepard AD, Weaver WD. Cost analysis of endovenous catheter ablation versus surgical stripping for treatment of superficial venous insufficiency and varicose vein disease. J Vasc Surg Venous Lymphat Disord. 2014;2:98-103.

11. Sutzko DC, Andraska EA, Obi AT, Sadek M, Kabnick LS, Wakefield TW, MD, et al. Age is not a barrier to good outcomes after varicose vein procedures. J Vasc Surg Venous Lymphat Disord. 2017;5:647-57.

12. Weinmann EE, Poluksht N, Chayen D, Bass A. Surgery of the Superficial Venous System in Elderly Patients. Vasc Endovascular Surg. 2003;37(2):111-5.

13. Gang C, Han-Cheng G, Ji-Ting W, Qiang H, Jian-Chun C. Comparison of endovenous laser treatment and high ligation in treatment of limb varicosity: A meta-analysis. Int Wound J. 2019;16(3):696.

14. Canonico S, Campitiello F, Santoriello A. Feasibility and Problems of Day-Care Varicose Vein Surgery in Elderly patients. J Ambulatory Surg. 2003;10:163-6.

15. Tzilins A, Salles-Cunha SX, Dosick SM, Gale SS, Seiwert AJ, Comerota AJ. Chronic Venous Insufficiency Due to Great Saphenous Vein Incompetence Treated with Radiofrequency Ablation: An Effective and Safe Procedure in the Elderly. Vasc Endovascular Surg. 2005;39(4):341-5.

16. Rivzi SA, Hingorani A, Kibrik P, Patel R, Victory J, Rybitskiy D, et al. Is Endovenous Heat-Induced Thrombosis Bialteral? J Vasc Surg. 2016;64(3):839.

17. Sufian S, Arnez A, Labropoulos N, Lakhanpal S. Incidence, progression, and risk factors for endovenous heat-induced thrombosis after radiofrequency ablation. J Vasc Surg Venous Lymphat Disord. 2013;1(2):159-64.

18. Kibrik P, Chait J, Arustamyan M, Alsheekh A, Rajaee S, Marks N, et al. Safety and efficacy of endovenous ablations in octogenarians, nonagenarians, and centenarians. J Vasc Surg Venous Lymphat Disord. 2020;8(1):95-9.

19. Kabnick L, Ombrellino M, Agis H. Endovenous heat induced throm- bus (EHIT) at the superficial-deep venous junction: a new post-treat- ment clinical entity, classification and potential treatment strategies. 18th Annual Meeting of the American Venous Forum. Miami, FL, USA; febrero 2006.

20. Bootun R, Lane TRA, Davies AH. The advent of non-thermal, non-tumescent techniques for treatment of varicose veins. Phlebology. 2016;31(1):5-14.

21. Brittenden J, Cooper D, Dimitrova M, Scotland G, Cotton SC, Elders A, et al. Five-Year Outcomes of a Randomized Trial of Treatments for Varicose Veins. N Engl J Med. 2019;381(10):912-22.

22. Hamel-Desnos C, Desnos P, Allaert FA, Kern P. Thermal Ablation of Saphenous Veins is Feasible and Safe in Patients Older than 75 Years: A Prospective Study (EVTA study). Phlebology. 2015;30(8):525-32. 